\title{
Collagen-Hydroxyapatite Microspheres as Carriers for Bone Morphogenic Protein-4
}

\author{
*Yng-Jiin Wang, †Feng-Huei Lin, $\neq$ Jui-Sheng Sun, †Yi-Chau Huang, \\ $\S$ Shan-Chang Chueh, and §Fu-Yin Hsu
}

\begin{abstract}
*Institute of Biomedical Engineering, National Yang-Ming University; †Institute of Biomedical Engineering, National Taiwan University; Department of Orthopedic Surgery, National Taiwan University Hospital, Taipei, Taiwan, ROC; and \$Material Research Laboratories, Industrial Technology Research Institute, Chutung, Hsinchu, Taiwan, ROC
\end{abstract}

\begin{abstract}
The purpose of the current study is to evaluate the carrier capability of collagen-hydroxyapatite microspheres to the bone morphogenic proteins (BMP). After anesthesia, a bone defect $(6.0 \mathrm{~mm}$ in diameter and $10.0 \mathrm{~mm}$ in depth) was created at the distal femoral condyles of New Zealand white rabbits. These $10.0 \mathrm{~mm}^{3}$ defects were then completely filled with the implant materials. After 2, 4, 6, and 8 weeks, the animals were sacrificed and histological evaluations were performed. The results showed that when the defects were left untreated, there was no evidence of bone formation during the eight-week experimental period. In the group treated with collagen-hydroxyapatite microspheres without BMP-4, the defect was filled with
\end{abstract}

fibrous tissue and inflammatory cells, while active bone formation with mature marrow tissue formation was evident in the defect treated with collagen-hydroxyapatite microspheres containing BMP-4. Collagen-hydroxyapatite microspheres were expected to be replaced by the regenerated bone structure as the bone reconstruction and bone remodelling process occurred. It was apparent that bone regeneration was influenced by the addition of BMP-4. Collagen-hydroxyapatite microspheres were good carriers for bone morphogenic proteins. Key Words: Collagen-Hydroxyapatite-Microspheres-Bone morphogenic protein-4-Bone defect.
The formation and repair of bone defect requires a source of pluripotential mesenchymal stem cells capable of differentiating into osteoblasts (1); growth factors that direct these cells to migrate into the osseous defect (2); a matrix or scaffold, to support the attachment and migration of these cells into the osseous defect (3); and the formation of a vascular network throughout the newly formed bone (4). However, the capacity of the human body to generate bone components is limited. Various biomaterials have been studied as tissue replacements for bone defects. The commonly used bone grafting materials included autografts (5), allografts (6), xenografts, and synthetic composites (7). Although autografts are most suitable for bone regeneration, they have to be dissected from the other bone tissues of the host; this

Received October 2001; revised March 2002.

Address correspondence and reprint requests to Dr. Jui-Sheng Sun, Department of Orthopedic Surgery, National Taiwan University Hospital, No. 7, Chung-Shan South Road, Taipei, Taiwan, ROC. E-mail: drjssun@ccms.ntu.edu.tw causes donor site morbidity and it is usually difficult to form the desired shape. Moreover, the risks of autograft for all patients are longer operations and anesthesia, high blood loss, infection, damage to nerves and blood vessels, thrombosis, and fracture. Allografting and xenografting have the disadvantages of causing immune reactions and transferring pathogens, and regularly provoke an immunodefensive reaction, which limits their efficiency, application, and availability (8).

Collagen and hydroxyapatite have been used as bone-filling materials in orthopedic surgery (9). The collagen fibers are known to serve as the scaffold for tissue repair (10), whereas hydroxyapatite is compatible and osteoconductive for bone regeneration. Although the dense hydroxyapatite disc is not biodegradable, particulate hydroxyapatite can be removed and remodeled in the host (11). However, direct implantation of hydroxyapatite particles resulted in the dislocation of material within the tissue. To eliminate the undesired mobility, hydroxyapatite powders are often mixed with collagen, 
gelatin, or fibrin glue when used as bone grafts (12-14). Although most of the collagen matrices are prepared in a slab form, spherical composites of collagen and hydroxyapatite are more versatile in clinical applications. Other than being used for cell culturing, spherical gel beads have closer packing and greater flexibility in filling different geometric cavity than do the nonspherical shapes. Smaller microspheres composed of collagen and hydroxyapatite are also potentially injectable for repairing tissue defects. In the pilot study of our institute, we demonstrated that the microgel beads served as good supports for the growth of osteoblast cells and did not induce immune response when particulate hydroxyapatites dispersed in the reconstituted atelocollagen matrix (15). In this study, we used these newly designed microspheres of hydroxyapatite/reconstituted collagen as supports for osteoblast cell growth.

One might expect the direct implantation of progenitor cells could lead to more rapid, uniform, and reliable healing of bone defects. A major challenge to this approach has been the identification of the proper type and source of cells for autologous cell therapy. Several experimental approaches have been utilized to elicit the formation of bone in segmental bone defects and to promote their healing. These approaches include the implantation of osteoconductive extracellular-matrix scaffolds (16) and the implantation of bone morphogenic proteins in various matrices $(17,18)$. The purpose of the current study was to evaluate the ability of collagenhydroxyapatite containing microspheres as a carrier for the bone morphogenic protein to repair a critical-sized defect. A model for the defect at the rabbit's distal femur was used, and the defect was left untreated, was filled with collagenhydroxyapatite microspheres, or was filled with collagen-hydroxyapatite microspheres containing bone morphogenic protein-4. Bone formation and healing at the site of the defect were evaluated with the use of radiographic and histological evaluation.

\section{MATERIALS AND METHODS}

\section{Preparation of implanted materials}

Hydroxyapatite was purchased from Merck (Darmstadt, Germany) and further characterized in the laboratory of National Yang-Ming University, Taipei, Taiwan. Collagen was prepared from rat tail tendon, as previous described (15). In brief, type I collagen was extracted from rat tail tendon, then soaked in $0.5 \mathrm{M}$ acetic acid for 3-4 days at $4^{\circ} \mathrm{C}$. Pepsin $(0.5 \mathrm{mg} / \mathrm{ml}$ in $0.5 \mathrm{M}$ acetic acid) was added subsequently and the solution incubated for $24 \mathrm{~h}$. The insoluble material was removed from the preparation by centrifuge at $12,000 \mathrm{rpm}$ for $1 \mathrm{~h}$ at $4^{\circ} \mathrm{C}$. $\mathrm{NaCl}$ was then added to the pepsin-soluble portion to a final concentration of $1 \mathrm{M}$. After standing overnight, the mixture was centrifuged at $12,000 \mathrm{rpm}$ and the precipitate was collected, resuspended in $0.5 \mathrm{M}$ acetic acid. Finally, the collagen solution was dialyzed against $0.5 \mathrm{M}$ acetic acid at $4^{\circ} \mathrm{C}$ to remove salt. The collagen was stored in $0.5 \mathrm{M}$ acetic acid at $4^{\circ} \mathrm{C}$.

Microspheres composed of particulate hydroxyapatite (particle size $5.32 \mu \mathrm{m}$ ) dispersed in fibrous collagen matrices were prepared as described previously (15). In brief, the procedure involved the droplet formation of hydroxyapatite/collagen mixture emulsified in olive oil, followed by the reconstitution of collagen in the presence of hydroxyapatite particles at $37^{\circ} \mathrm{C}$. Microspheres sized at $600-700 \mu \mathrm{m}$ could be obtained by controlling the stirring speed of the emulsified mixture at $400 \mathrm{rpm}$, when $2 \%$ Span 85 was present in the emulsion mixture. Then the mid-products were freeze-dried to get final products used in this experiment. Such prepared microspheres will absorb and swell when mixed with BMP-4 solution. The microspheres thus obtained can be used as carriers of BMP-4 to support the growth of osteoblast cells.

\section{Operation and implantation}

New Zealand white rabbits (male, weight: 2.5$3.0 \mathrm{~kg}$ ) were used in the study. The animals were fed Purina Laboratory Chow ad libitum and housed in a temperature-, humidity-, and light-controlled environment. Surgical procedures and experimental protocols were approved and under supervision by the Medical College's Animal Research Committee of the National Taiwan University. The rabbits were anesthetized by ketamine $(25 \mathrm{mg} / \mathrm{kg}$, s.c., Sintong, Taiwan, ROC) and Combelen (N-(3'-dimethylaminopropyl)-3-propionylphenothiazine) $(5 \mathrm{mg} / \mathrm{kg}$, s.c., Bayer AG, Leverkusen, England) and local administration of $0.5 \%$ lidocaine. After shaving, disinfecting, and sterile draping of the operation site, the femoral condyles were exposed by lateral longitudinal incision. Initially, a bone defect was created by a $3.2 \mathrm{~mm}$ drill and was subsequently enlarged with a $6 \mathrm{~mm}$ drill. All the drill holes were carefully rinsed with Ringer's solution so that any abraded particles formed during drilling were removed. These defects were then completely filled with the implant materials. The periosteum, fascia, and skin were sutured layer by layer. After 2, 4, 6, and 8 weeks, the animals were sacrificed with an overdose of intravenous pentobarbital. A total of 24 rabbits divided into two 
groups for the above experimental time periods were used in the study. In the group I, the left side was shaved and operated without insertion of any material, while collagen-hydroxyapatite microspheres were implanted in the right side before the closure of the wound. In the left side of group II rabbits, the collagen-hydroxyapatite microspheres without bone morphogenic protein were implanted before the closure of the wound; while in the right side, collagen-hydroxyapatite microspheres containing $10 \mathrm{~g}$ human bone morphogenic protein-4 (BMP-4, R \& D Systems, Inc., Minneapolis, MN, U.S.A.) were inserted into the bone defect. The animals were allowed to recover from anesthesia, and were placed into cages until the end of the experiment.

\section{Radiographic analysis and histological evaluations}

After harvesting the specimens, the lateral radiographs of the femur were made and analyzed. The hindlimbs were harvested from the treated animals at the mentioned time periods after operation. Implants and surrounding tissues were removed en bloc, washed in normal saline and fixed with $4 \%$ formaldehyde in phosphate buffered solution for 18 hours, decalcified, dehydrated in alcohol, cleared in xylene, and embedded in paraffin. Four sections ( 5 to $7 \mu \mathrm{m}$ in thickness) parallel to the major axis were cut for each implant and stained with hematoxylin and eosin. Each section was carefully observed, and representative findings were photographed by using light microscope.

\section{RESULTS}

\section{Radiographic analysis}

The operated limbs did not show any radiographic change. The HA microsphere implants with or without BMP-4 cells could be easily visualized because of the radio-opacity of the implanted material (Fig. 1). Immediately postoperatively, the implants had a granular appearance; as new bone formed, the appearance of the implant became smaller and more radiolucent. The appearance of this radiolucency was considered to be an indication of resorption of the implant.

\section{Histological evaluation}

The formation of new bone in the empty cavities (control group) was restricted to the edge of the defect. The large part at the center of the defect remained free of bone and was filled with bone marrow rich in fat cells at the end of the 8-week test (Fig. 2). When the results obtained were evaluated as a function of time, it became apparent that progres-


FIG. 1. Radiographs are shown of a bone defect that had been left untreated (control) and had been treated with a collagenhydroxyapatite microsphere (HA-loaded). In the bone defect treated with a collagen-hydroxyapatite microsphere, the implants had a granular appearance (arrows); as new bone formed, the appearance of the implant became smaller and more radiolucent.

sive bony reconstruction of the defect cavity with the course of time was not detected.

The formation of new bone within the metaphyseal defect was not observed in the group treated with collagen-hydroxyapatite microspheres without BMP-4 addition. The large part at the center of the defect remained free of bone during the entire course of the test up to 8 weeks, and was filled with fibrous tissue and inflammatory cells infiltration. The implanted collagen fibers were still visible at this

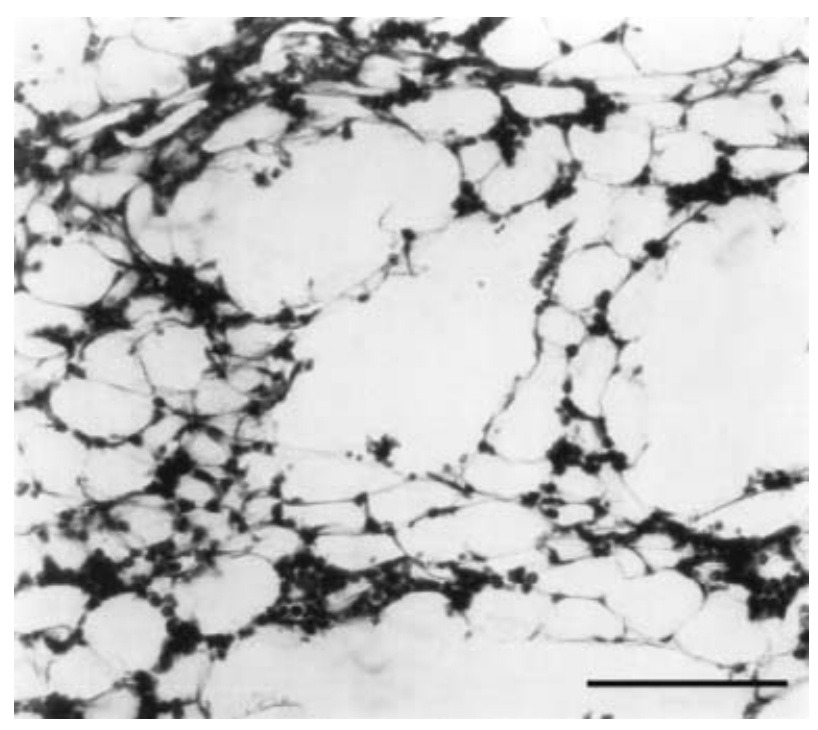

FIG. 2. The photomicrograph, made at eight weeks, shows a representative histological section from an untreated defect. $(\mathrm{H} . \mathrm{E}$. Stain; Bar $=100 \mu \mathrm{m})$ The formation of new bone in the empty cavities was restricted to the edge of the defect. The large part at the center of the defect remained free of bone and was filled with bone marrow rich in fat cells. 


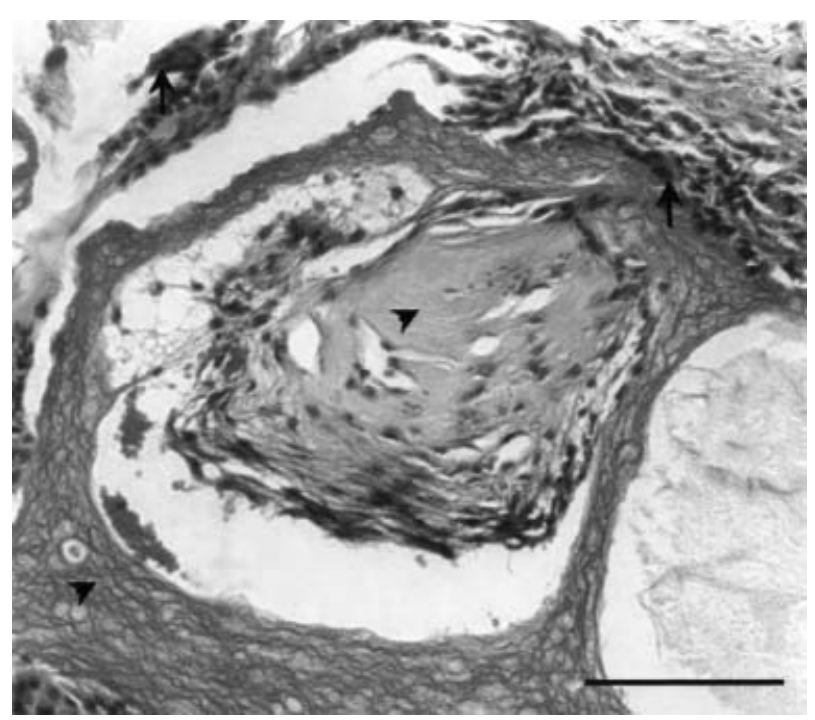

FIG. 3. The photomicrograph, made at eight weeks, shows a representative histological section with bone defect treated with collagen-hydroxyapatite microspheres without bone morphogenic protein. (H.E. Stain; Bar $=100 \mu \mathrm{m}$ ) The formation of new bone within the metaphyseal defect was not observed in this group. The implanted collagen fibers were still visible at this stage and showed evidence of degeneration (arrow heads). The large part at the center of the defect remained free of bone, and was filled with fibrous tissue and inflammatory cells infiltration. The inflammatory cells infiltration with giant cells formation (arrows) were apparent at this stage.

stage and showed evidence of degeneration. When the results obtained were evaluated as a function of time, it appeared that the inflammatory cells infiltration with giant cells formation became more apparent as the time elapsed (Fig. 3). In other words, progressive increased inflammatory reaction within the defect cavity was detected.

Active new bone formation was evident in the group treated with collagen-hydroxyapatite microspheres containing BMP-4. In the second week postoperatively, the defective cavity was filled with a hematoma and connective tissue (Fig. 4), and the giant cells formation were quite evident at this stage. At the fourth week after operation, immature woven bone was found in the defect and the defect was surrounded with active osteoblasts. The giant cells formation were still quite evident, and there were numerous neovascularization formed at this stage (Fig. 5). Figure 6 shows the results of histological examination at 6 weeks; the breakdown and dissolution of the collagen-hydroxyapatite microspheres were observed in the histological sections at this stage. The regenerated bone was vigorously growing into the interspaces of the implanted material (Fig. 6). In the eighth week after operation, the implant gradually dissolved and was replaced by the bony



FIG. 4. The photomicrograph, made at two weeks, shows a representative histological section which was bone defect treated with collagen-hydroxyapatite microspheres with bone morphogenic protein. (H.E. Stain; Bar $=100 \mu \mathrm{m}$ ) The defective cavity was filled with a haematoma (arrow head) and connective tissue, and the inflammatory cells infiltration with giant cells formation (arrows) were quite obvious.

structure (Fig. 7). The laminar bone appeared, and the collagen-hydroxyapatite microspheres continued to be dissolved, digested, and replaced by the physiological bony marrow tissue (Fig. 7). It was apparent

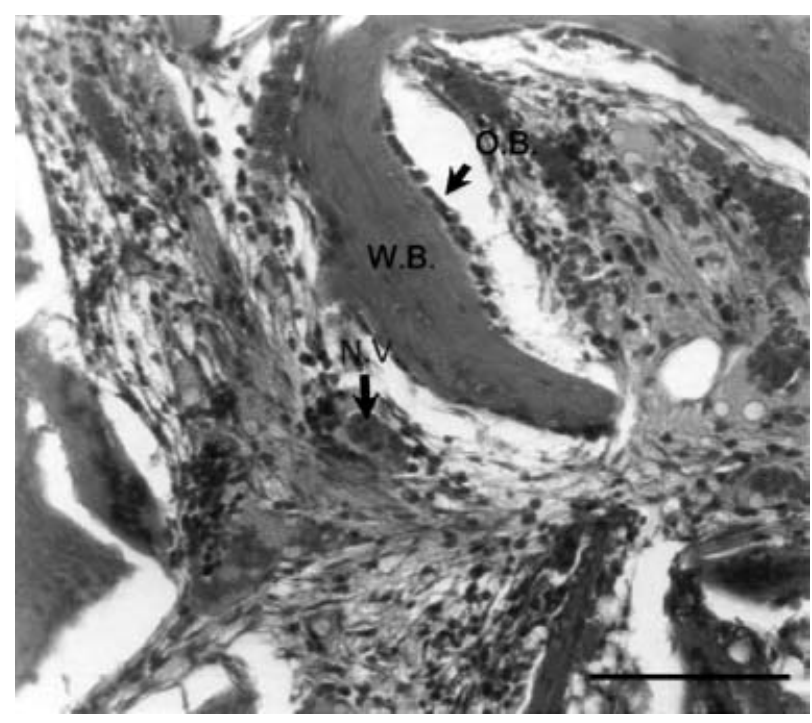

FIG. 5. The photomicrograph, made at four weeks, shows a representative histological section which is bone defect treated with collagen-hydroxyapatite microspheres with bone morphogenic protein. (H.E. Stain; Bar $=100 \mu \mathrm{m})$ After the fourth week postoperation, immature woven bone (W.B.) was found in the defect cavity, which was surrounded with active osteoblasts (O.B.) proliferation. The inflammatory cells infiltration with giant cells formation were quite evident, and there were numerous neovascularization (N.V.) formed at this stage. 


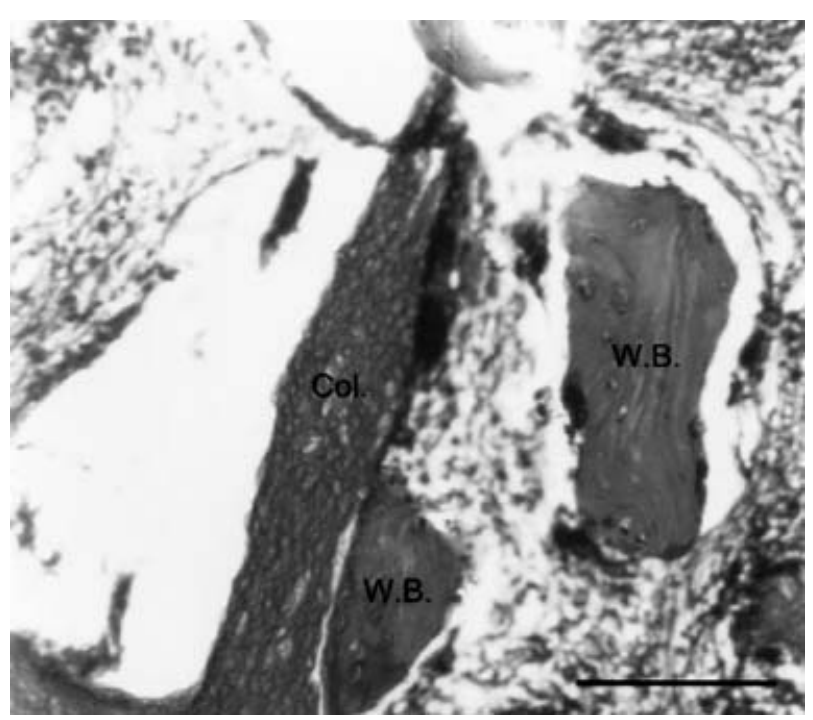

FIG. 6. The photomicrograph, made at six weeks, is of a representative histological section which is bone defect treated with collagen-hydroxyapatite microspheres with bone morphogenic protein. (H.E. Stain; Bar $=100 \mu \mathrm{m})$ The breakdown and dissolution of the collagen-hydroxyapatite microspheres (Col.) were observed in the histological sections at this stage. The regenerated bone (W.B.) was vigorously growing into the interspaces of the implanted material.

that bone regeneration was occurring in connection with, and influenced by the BMP-4. Collagenhydroxyapatite microspheres were expected to be progressively phased out in the physiochemical environment and subsequently replaced by the regenerated bone structure as the bone reconstruction and bone remodeling process occurred.

\section{DISCUSSION}

For many years, bone grafts have been used by orthopedic surgeons to aid in the repair of recalcitrant bone damage. Lacroix hypothesized that bone contains a substance, osteogenin, that initiates bone growth (19). Urist made the key discovery that demineralized, lyophilized, segments of rabbit bone can induce new bone formation (20). Extensive characterization of this process indicates that it mirrors the normal process of in vivo cartilage and bone formation (21). Initially, the implant is invaded by migrating mesenchymal cells, which differentiate over a period of approximately 7 days into cartilage forming cells (chondroblasts and chondrocytes). Bone cells (osteoblasts and osteoclasts) then appear in the area, and are responsible for the gradual removal of cartilage and the deposition of new bone in its place. The end result is the replacement of the originally implanted material with new bone and functional marrow. Later, the active proteinaceous component in the bone extract was identified and named as bone morphogenic protein (BMP) by Urist and collaborators (22).

The design and selection of an ideal carrier for the delivery of BMP is based on several criteria. First, the material should allow for uniform bony growth and retention of bone cells. Second, the carrier should support rapid vascular ingrowth. Third, the matrix should be composed of radiolucent materials that are resorbed and replaced by bone as new bone is formed. Fourth, the material should allow or enhance osteoconductive bridging of host bone by the new bone. Finally, the cell-matrix combination should be easy for the physician to handle in a clinical setting. The form of ceramic (hydroxyapatite) that was used in the present study may not be the ideal delivery vehicle for the treatment of bone defects, because the radiopacity and slow resorption of this ceramic may make it difficult for new bone to be distinguished radiographically from the original ceramic; but the completely interconnected collagen fiber microspheres will compensate for this deficit. A comparison of insoluble collagen, hydroxyapatite, tricalcium phosphate, glass beads, and polymethyl-methacrylate as carriers has revealed collagen to be the optimal delivery system for BMPs (23). In the present study, we use the collagen-hydroxyapatite microspheres as a carrier system for BMPs.

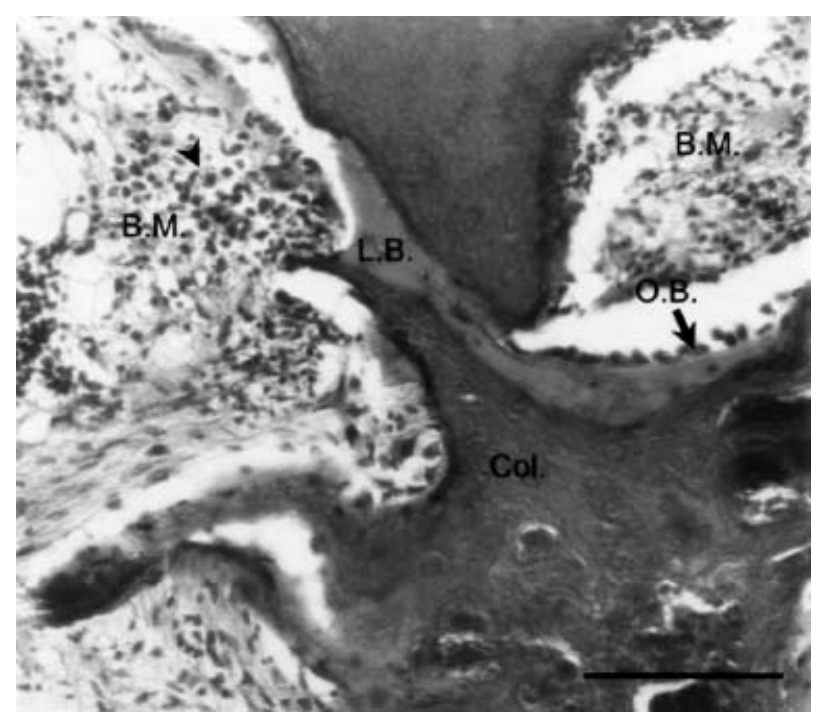

FIG. 7. Photomicrograph, made at eight weeks, of a representative histological section which is bone defect treated with collagen-hydroxyapatite microspheres with bone morphogenic protein. (H.E. Stain; Bar $=100 \mu \mathrm{m})$ In this stage, the implant gradually dissolved and was replaced by the bony structure. The laminar bone (L.B.) appeared, and the collagen-hydroxyapatite micropsheres (Col.) continued to be dissolved, digested, and replaced by the physiological bony marrow tissue (B.M.) that was filled with active bone marrow cells (arrow head). 
In certain species, hydroxyapatite alone appears to be "osteoinductive" (24). For example, in subhuman primates, hydroxyapatite alone induces bone, albeit at a much slower rate than when in combination with BMPs. One interpretation is that osteoinductive endogenous BMPs in circulation progressively bind to the implanted disc of hydroxyapatite. When an optimal threshold concentration of native BMPs is achieved, the hydroxyapatite become osteoinductive. In this study, we used collagen-hydroxyapatite microspheres of granules sizes $600-700(\mu \mathrm{m})$ as the BMP delivery system in determining efficiency of bone induction. This study revealed that in rabbits, microspheres containing BMP-4 were consistently osteoinductive, whereas microspheres without BMP-4 in the same manner were inactive. New bone formed throughout the entire network of holes in the implants that had been loaded with BMP-4 (Figs. 4-7). No such collar formed around any of the implants that had not been loaded with BMP-4, and there was no new bone formation along the host bone that had been left untreated (Figs. 2 and 3). The results of the present study demonstrate that the implantation of BMP-4 can induce new-bone formation in the $10.0 \mathrm{~mm}^{3}$-sized bone defects.

Bone morphogenic protein induced bone defect healing in this study is an extremely complex process. The first of the three predominant stages of fracture healing is the inflammatory phase, in which the hematoma is formed from the blood vessels ruptured by the injury. Soon afterward, inflammatory cells invade the clot and initiate the lysosomal degradation of necrotic tissue (Fig. 4). In the normal progression of healing, the second, or reparative phase, begins before 4 weeks of injury, overlapping the end stages of inflammation. The reparative phase is characterized by the invasion of pluripotential mesenchymal stem cells, which differentiate into fibroblasts and osteoblasts. This diverse population of cells is responsible for the formation of a soft fracture callus and its subsequent transition to woven bone (Fig. 5). The concurrent proliferation of blood vessels (angiogenesis) within tissues and marrow space helps to route the appropriate cells to the defect site, and contributes to the formation of a bed of granulation tissue (Fig. 5). The final and longer phase of healing, which overlaps significantly with the reparative phase, is characterized by the slow modeling and remodeling of the fracture callus from woven to mature lamellar bone (Figs. 6 and 7), and ultimately, the restoration of the bone to normal or near normal morphology and mechanical strength. It was apparent that collagen-hydroxyapatite microspheres were expected to be progressively phased out in the physiochemical environment and subsequently replaced by the regenerated bone structure as the bone reconstruction and bone remodeling process occurred.

A number of investigators have studied the ability of BMPs to serve as a bone-grafting agent in the treatment of bone defects (25). Although some of these investigations have indicated that BMP is capable of promoting new-bone formation, techniques for enriching the active component of BMP are of primary importance. In a direct comparison of the osteogenic potential of collagen-hydroxyapatite microspheres with that of BMPs, we demonstrated that, with a ceramic carrier, BMP-4 promoted faster and more extensive new-bone formation than collagen-hydroxyapatite microspheres did. We attributed this finding to the addition of BMP-4. The approach described in the present report offers the extraordinary advantage of the delivery of BMP-4 at local sites where repair or regeneration of tissue is needed. This is especially important in the clinical conditions in which the augmentation of bone is needed in order to overcome the natural decline in the regenerative capacity of tissue.

In summary, the present study established the technical feasibility of using implants loaded with BMPs to achieve site-specific new-bone formation in bone defects. This technique may prove to be useful in association with a wide variety of clinical applications, including repair of segmental defects, spinal arthrodesis, fixation of prosthetic implants, and restoration of maxillofacial bone defect.

\section{REFERENCES}

1. Bab I, Gazit D, Muhlrad A, Shteyer A. Regenerating bone marrow produces a potent growth-promoting activity to osteogenic cells. Endocrinology 1988;123:345-52.

2. Bruder SP, Fink DJ, Caplan AI. Mesenchymal stem cells in bone development, bone repair, and skeletal regeneration therapy. J Cell Biochem 1994;56:283-94.

3. Bruder SP, Jaiswal N, Haynesworth SE. Growth kinetics, self-renewal, and the osteogenic potential of purified human mesenchymal stem cells during extensive subcultivation and following cryopreservation. J Cell Biochem 1997;64:27894.

4. Bruder SP, Horowitz MC, Mosca JD, Haynesworth SE. Monoclonal antibodies reactive with human osteogenic cell surface antigens. Bone 1997;21:225-35.

5. Beirne OR. Comparison of complications after bone removal from lateral and medial plates of the anterior ilium for mandibular augmentation. Int J Oral Maxillofac Surg 1986;15:269-72.

6. Mankin HJ, Gebhardt MC, Tomford WW. The use of frozen cadaveric allografts in the management of patients with bone tumors of the extremities. Orthop CIin North Am 1987;18: 275-89.

7. Costantino PD, Friedman CD. Synthetic bone graft substitutes. Otolaryngol Clin North Am 1994;27:1037-74.

8. Stupp SI, Hanson JA, Eurell JA, Ciegler GW, Johnson A. 
Organoapatites: materials for artificial bone. III. Biological testing. J Biomed Mater Res 1993;27:301-11.

9. Bell R, Beirne OR. Effect of hydroxylapatite, tricalcium phosphate, and collagen on the healing of defects in the rat mandible. J Oral Maxillofac Surg 1988;46:589-94.

10. Pachence JM. Collagen-based devices for soft tissue repair. J Biomed Mater Res 1996;33:35-40.

11. Pohunkova H, Adam M. Reactivity and the fate of some composite bioimplants based on collagen in connective tissue. Biomaterials 1995;16:67-71.

12. Sugaya A, Minabe M, Tamura T, Hori T. Effects on wound healing of hydroxyapatite-collagen complex implants in periodontal osseous defects in the dog. $J$ Periodontal Res 1989;24:284-8.

13. Nagase M, Chen R, Asada Y, Nakijima T. Radiographic and microscopic evaluation of subperiosteally implanted blocks of hydroxyapatite gelatin mixture in rabbits. J Oral Maxillofac Surg 1989;47:40-5.

14. Wittkampf AR. Augmentation of the maxillary alveolar ridge with hydroxyapatite and fibrin glue. J Oral Maxillofac Surg 1988;46:1019-21.

15. Hsu FY, Chueh SC, Wang YJ. Microspheres of hydroxyapatite/reconstituted collagen as supports for osteoblast cell growth. Biomaterials 1999;20:1931-6.

16. Hollinger JO, Brekke J, Gruskin E, Lee D. Role of bone substitutes. Clin Orthop 1996;324:55-65.
17. Gerhart TN, Kirker-Head CA, Kriz MJ, Holtrop ME, Hennig GE, Hipp J, Schelling SH, Wang E. Healing segmental femoral defects in sheep using recombinant human bone morphogenetic protein. Clin Orthop 1993;293:317-26.

18. Mayer M, Hollinger J, Ron E, Wozney J. Maxillary alveolar cleft repair in dogs using recombinant human bone morphogenetic protein-2 and a polymer carrier. Plast Reconstr Surg 1996;98:247-59.

19. Lacroix P. Recent investigations on the growth of bone. Nature 1945; $156: 576$.

20. Urist MR. Bone: formation by autoinduction. Science 1965;150:893-9.

21. Reddi AH. Cell biology and biochemistry of endochondral bone development. Collagen Relat Res 1981;1:209-26.

22. Urist MR, Mikulski A, Lietze A. Solubilized and insolubilized bone morphogenetic protein. Proc Natl Acad Sci USA 1979;76:1828-32.

23. Ma S, Chen G, Reddi AH. Collaboration between collagenous matrix and osteogenin is required for bone induction. Ann $N$ Y Acad Sci 1990;580:524-5.

24. Ripamonti U. Osteoinduction in porous hydroxyapatite implanted in heterotopic sites of different animal models. Biomaterials 1996;17:31-5.

25. Reddi AH, Huggins C. Biochemical sequences in the transformation of normal fibroblasts in adolescent rats. Proc Natl Acad Sci USA 1972;69:1601-5. 